\title{
Presurgical EEG-fMRI in a complex clinical case with seizure recurrence after epilepsy surgery
}

This article was published in the following Dove Press journal:

Neuropsychiatric Disease and Treatment

25 July 2013

Number of times this article has been viewed

\author{
Jing Zhang' \\ Qingzhu Liu \\ Shanshan $\mathrm{Mei}^{2}$ \\ Xiaoming Zhang ${ }^{2}$ \\ Xiaofei Wang ${ }^{2}$ \\ Weifang Liu' \\ Hui Chen' \\ Hong Xia' \\ Zhen Zhou' \\ Yunlin $\mathrm{Li}^{2}$ \\ 'School of Biomedical Engineering, \\ Capital Medical University, Beijing, \\ People's Republic of China; \\ ${ }^{2}$ Department of Functional \\ Neurology and Neurosurgery, \\ Beijing Haidian Hospital, Beijing, \\ People's Republic of China
}

Correspondence: Jing Zhang School of Biomedical Engineering, Capital Medical University, Beijing 100069, People's Republic of China

Tel $+8610839 \mid 1543$

Fax +861083911544

Email jzhang0000@।63.com
Abstract: Epilepsy surgery has improved over the last decade, but non-seizure-free outcome remains at $10 \%-40 \%$ in temporal lobe epilepsy (TLE) and 40\%-60\% in extratemporal lobe epilepsy (ETLE). This paper reports a complex multifocal case. With a normal magnetic resonance imaging (MRI) result and nonlocalizing electroencephalography (EEG) findings (bilateral TLE and ETLE, with more interictal epileptiform discharges [IEDs] in the right frontal and temporal regions), a presurgical EEG-functional MRI (fMRI) was performed before the intraoperative intracranial EEG (icEEG) monitoring (icEEG with right hemispheric coverage). Our previous EEG-fMRI analysis results (IEDs in the left hemisphere alone) were contradictory to the EEG and icEEG findings (IEDs in the right frontal and temporal regions). Thus, the EEG-fMRI data were reanalyzed with newly identified IED onsets and different fMRI model options. The reanalyzed EEG-fMRI findings were largely concordant with those of EEG and icEEG, and the failure of our previous EEG-fMRI analysis may lie in the inaccurate identification of IEDs and wrong usage of model options. The right frontal and temporal regions were resected in surgery, and dual pathology (hippocampus sclerosis and focal cortical dysplasia in the extrahippocampal region) was found. The patient became seizure-free for 3 months, but his seizures restarted after antiepileptic drugs (AEDs) were stopped. The seizures were not well controlled after resuming AEDs. Postsurgical EEGs indicated that ictal spikes in the right frontal and temporal regions reduced, while those in the left hemisphere became prominent. This case suggested that (1) EEGfMRI is valuable in presurgical evaluation, but requires caution; and (2) the intact seizure focus in the remaining brain may cause the non-seizure-free outcome.

Keywords: EEG-fMRI, focus localization, presurgical evaluation, epilepsy surgery

\section{Introduction}

Epilepsy surgery has improved over the last decade, approaching $60 \%$ to $90 \%$ seizure-free outcome in patients with temporal lobe epilepsy (TLE) and $40 \%$ to $60 \%$ in extratemporal lobe epilepsy (ETLE). ${ }^{1}$ However, non-seizure-free outcome remains nonignorable. Major causes of non-seizure-free outcome have been identified, and incomplete or nonresection of unrecognized seizure foci usually results in unfavorable seizure control. ${ }^{2}$

Precise localization of the epileptic foci is a prerequisite for good surgical outcome, but it remains a challenge, especially for ETLE and nonlesional epilepsy which has normal magnetic resonance imaging (MRI). Simultaneous electroencephalography (EEG) and functional MRI (fMRI) opens the opportunity to uncover the possible generators of interictal epileptiform discharges (IEDs). ${ }^{3-9}$ Currently, the clinical utility of EEG-fMRI is still unclear, and a number of studies that compared EEG-fMRI findings 
with those of the intracranial EEG (icEEG) in epilepsy presurgical evaluation have obtained varied findings. ${ }^{3-6,10-19}$ Al Asmi et al found that icEEG confirmed 50\% (4/8) of the EEG-fMRI findings. ${ }^{4}$ Bagshaw et al reported that EEG-fMRI results were $75 \%(3 / 4)$ consistent with those of icEEG. ${ }^{5}$ Zijlmans et al studied patients who were rejected for epilepsy surgery because of nonlocalized seizure focus and showed that EEG-fMRI improved localization in 66.7\% (4/6) of unclear focal foci, and icEEG supported the EEG-fMRI results in $50 \%(2 / 4)$ of the patients. ${ }^{11}$ Grova et al demonstrated that the EEG-fMRI results were concordant with icEEG results in $100 \%$ (3/3) patients. $^{13}$ Grouiller et al improved the EEGfMRI approach and found EEG-fMRI to be concordant or moderately concordant with the icEEG and/or surgical site, in $77.8 \%(14 / 18)$ of the patients. ${ }^{17}$

In this paper, we report a complex case of bilateral TLE (bi-TLE) and bi-ETLE that used EEG-fMRI for presurgical evaluation. In this case, normal MRI and nonlocalizing EEG findings made it difficult to localize the seizure foci in presurgical evaluation. In addition, the presurgical icEEG monitoring (with electrodes placed in both hemispheres) was skipped for financial reasons (the patient's family could not afford it). This made the surgical decision more difficult, and the surgical site was determined largely based on the findings of the presurgical EEG and intraoperative icEEG (covered the right frontal and temporal regions). However, the intraoperative icEEG findings could neither rule out an epileptogenic focus in the left hemisphere nor provide a full picture of IED spikes in both hemispheres. Therefore, inexpensive noninvasive neuroimaging, such as EEG-fMRI, was much needed for focus localization in this case. Unfortunately, our presurgical EEG-fMRI analysis failed (due to lack of experience in EEG-fMRI data processing and lack of expertise in IED identification); however, the data were reanalyzed recently and the causes of this failure were identified. Further, possible reasons for the non-seizure-free outcome of this case are discussed.

\section{Case report}

A 17-year-old right-handed patient with a history of refractory focal epilepsy for 8 years was studied. The patient had seizures several times a day. Multiple antiepileptic medications (AEDs) failed to control the frequent seizures.

A video (scalp) EEG recording yielded nonlocalizing results: the interictal EEG showed IED spikes in both hemispheres, especially in the anterior frontal lobe, and a large amount of IED spikes in the right frontal and temporal regions; ictal EEG showed abnormal spikes that were widespread in both hemispheres. In addition, structural MRI was normal. EEGfMRI was performed to aid the presurgical evaluation. Our presurgical EEG-fMRI analysis found major brain activation in the left hemisphere alone, contradictory to the findings of EEG and the later intraoperative icEEG. The right temporal and frontal regions were resected based on the icEEG findings.

The patient became seizure-free for 3 months after surgery, but seizures restarted after antiepileptic medications were stopped. The patient paid his first revisit to the hospital 4 months after surgery and was retreated with antiepileptic medications, which initially controlled the seizures. However, seizures recurred later and gradually became more frequent. The patient revisited the hospital again 16 months after surgery, and more medications were used for seizure control.

\section{Methods}

\section{Data acquisition}

The patient who participated in the study gave written informed consent, and this study was approved by the Institutional Review Board (IRB) at the Capital Medical University, Beijing, People's Republic of China. The EEG-fMRI scan was performed using a 32-channel MR-compatible EEG system (Brain Products, Munich, Germany) and a 3T MRI scanner (Siemens Trio, Erlangen, Germany). The EEG was first recorded outside the MRI scanner, for about 10 minutes, and then inside the scanner, for 16 minutes (for the first 12 minutes, the EEG was recorded with $\mathrm{AMRI}$, followed by a structural MRI sequence with no EEG, followed by another 4 minutes of EEG with $\mathrm{fMRI}$ ). The EEG was acquired at $5 \mathrm{kHz}$, using a BrainAmp MR-compatible amplifier (Brain Products, Munich, Germany) and synchronized with the MRI clock. The electrodes were mounted according to the standard 10-10 electrode placement system and equipped with $5 \mathrm{k} \Omega$ resistance. The site $\mathrm{FCz}$ was used as recording reference, and the ECG was recorded with $15 \mathrm{k} \Omega$ resistance.

MRI images were acquired with a standard birdcage head coil. Functional images were acquired using a T2*-weighted gradient-echo, echo-planar imaging (EPI) sequence (25 axial slices of $4 \mathrm{~mm}$ thick, with $1 \mathrm{~mm}$ gap; $\mathrm{TE}($ echo time $)=30 \mathrm{~ms} ; \mathrm{TR}$ (repetition time $)=2000 \mathrm{~ms}$; flip angle $=90$; matrix $=64 \times 64$; in-plane resolution $3.4 \times 3.4 \mathrm{~mm}^{2}$ ) simultaneously with the EEG recordings (first 360 volumes and later 120 volumes). The patient was instructed to rest in the scanner with eyes closed during the scan. A high-resolution T1-weighted anatomical scan (MP-RAGE, 176 sagittal slices; $\mathrm{TE}=3.37 \mathrm{~ms}$; $\mathrm{TR}=2560 \mathrm{~ms}$; flip angle $=7$; matrix $=256 \times 256$ ) was also acquired. 
In addition, intraoperative icEEG was performed at the beginning of the surgery, with $2 \times 8$ subdural contact strips covering the right frontal and temporal regions.

Further, the specimens obtained from epilepsy surgery were sent to the Department of Pathology, Beijing Haidian Hospital for histopathological examination after the surgery, and the histopathological findings were used to compare with neuroimaging and EEG results. A postsurgical video EEG was also performed 4 months and 16 months after the surgery to check the surgical outcome.

\section{Data analysis}

Artifact removal was performed with Brain Vision Analyzer 2.0 (Brain Products Gmbh) using average artifact subtraction methods to remove the gradient and pulse artifacts (ie, the artifacts time-locked to cardiac activity). The EEG was then band-pass filtered $(1-30 \mathrm{~Hz})$ to remove the remaining high-pass artifact and downsampled to $250 \mathrm{~Hz}$ after artifact removal. Visual inspection of the EEG and marking of the spikes was performed by five raters (and times), resulting in five sets of IED event onsets (Table 1). A section of the filtered EEG with identified IED is illustrated in Figure 1.
fMRI data were analyzed by SPM8 (statistical parametric mapping software) (http://www.fil.ion.ucl.ac.uk/spm/). They were realigned, registered to the Montreal Neurological Institute (MNI) template brain, spatially smoothed (with a Gaussian kernel of $8 \mathrm{~mm}^{3}$ FWHM [full width at half-maximum]) and temporally high-pass filtered (cutoff frequency: 1/128 Hz).

The fMRI data were analyzed with a general linear model (GLM). The IED event onsets were convolved with the default hemodynamic response function using default model parameters in SPM8. The fMRI analysis was performed nine times, with different combinations of IED onsets and model parameters (Table 1). The main differences in the options and parameters of these fMRI analyses are listed in Table 1. Figure 2 shows the design matrices of these fMRI analyses.

SPM $t$-test was performed to test the significance of the modeled hemodynamic response at each voxel. The four analyses in the middle of Table 1 (analyses 3-6) used a threshold at $\mathrm{T}=4.73(P<0.05)$, corrected for multiple comparisons and family-wise error (FWE) because many voxels survived this threshold; while analyses 1-2 and 7-9 used a threshold at $\mathrm{T}=2.34(P<0.01)$, uncorrected for multiple comparisons because few voxels could survive a

Table I The main parameters used in EEG-fMRI analyses

\begin{tabular}{|c|c|c|c|c|}
\hline $\begin{array}{l}\text { Analysis } \\
\text { no }\end{array}$ & IED onsets (in scans) & $\begin{array}{l}\text { Time derivative } \\
\text { (yes/no) }\end{array}$ & $\begin{array}{l}\text { I st order time } \\
\text { modulation } \\
\text { (yes/no) }\end{array}$ & $\begin{array}{l}\text { Model interactions } \\
\text { with Volterra } \\
\text { (yes/no) }\end{array}$ \\
\hline $\mathrm{I}$ & 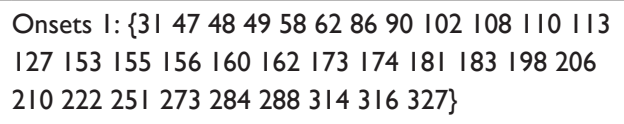 & Yes & Yes & Yes \\
\hline 2 & 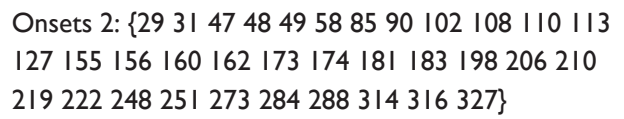 & Yes & Yes & Yes \\
\hline 3 & 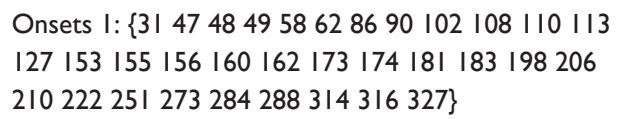 & No & No & No \\
\hline 4 & 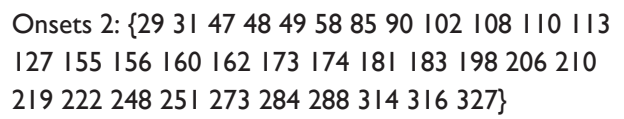 & No & No & No \\
\hline 5 & 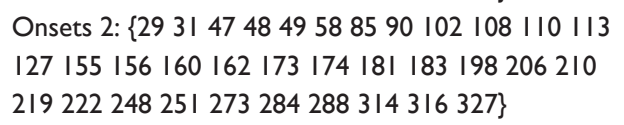 & Yes & No & No \\
\hline 6 & 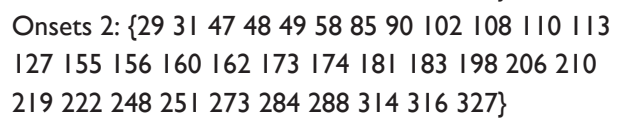 & Yes & Yes & No \\
\hline 7 & Onsets 3: $\{28263287\}$ & No & No & No \\
\hline 8 & Onsets 4: $\{28$ I02 181263287314$\}$ & No & No & No \\
\hline 9 & Onsets 5: $\{28$ I02 I8I 263287314 37| 416$\}$ & No & No & No \\
\hline
\end{tabular}

Notes: Onsets I-5: five sets of IED event onsets provided by five raters (and times). Analysis I: This analysis used the same IED onset and the same model options and parameters as those used in our presurgical EEG-fMRI analysis. Onsets 2-5 in analyses 2-9: IED onsets identified and used recently. IED onsets I-4 in analyses I-8 were marked for the first 360 volumes, and the last I20 volumes were ignored. IED onset 5 in analysis 9 was marked for all the 480 volumes.

Abbreviations: EEG, electroencephalography; fMRI, functional magnetic resonance imaging; IED, interictal epileptiform discharge. 


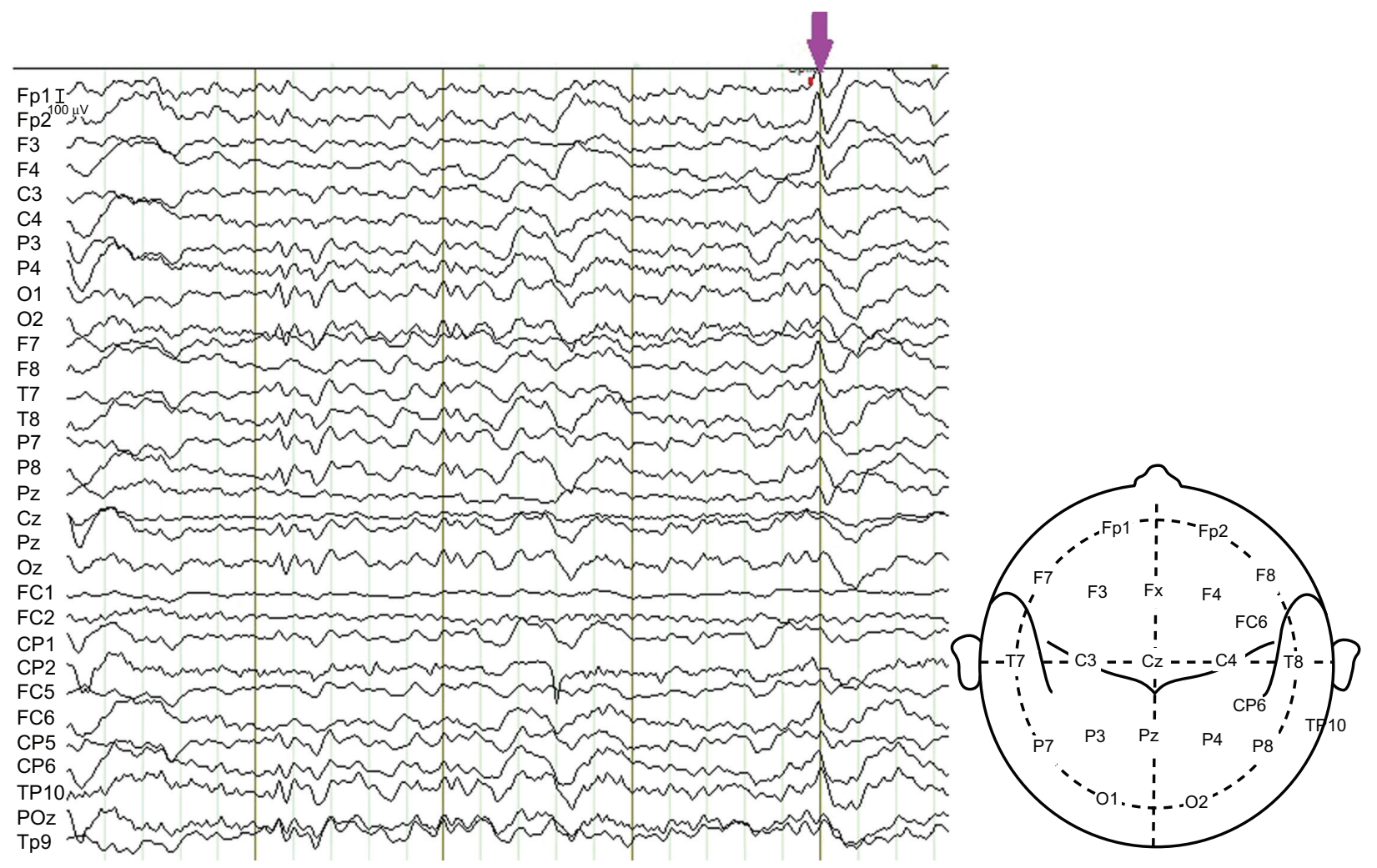

Figure I A section of the patient's EEG.

Notes: The EEG was recorded in the MRI room for EEG-fMRI acquisition and was filtered to remove the artifacts. An IED is marked by the arrow, which indicates that the abnormal spikes were mainly at the sites FPI, FP2, F4, T8, FC6 and TPI0.

Abbreviations: EEG, electroencephalography; fMRI, functional magnetic resonance imaging; IED, interictal epileptiform discharge; MRI, magnetic resonance imaging.

higher threshold. In addition, EEG-fMRI concordance to the locations of seizure foci was evaluated by comparing the locations of IED-related clusters (that survived threshold) in EEG-fMRI results with those of the surgical sites (detected by intraoperative icEEG).

\section{Results}

The results of the first and second analyses indicated that weak IED-related activation $(\mathrm{T}=2.34)(P<0.01$ [uncorrected] $)$ was in the left frontal and temporal regions (Figure 3 ). The next four fMRI analyses demonstrated relatively strong $(\mathrm{T}=4.73)$ $(P<0.05$ [FWE corrected]), IED-related activation in the right frontal region. In addition, the seventh analysis showed relatively weak activation in the right temporal region, and the eighth and ninth analyses revealed activation in the temporal and frontal regions in both hemispheres (Figure 3).

Intraoperative icEEG (with electrodes placed in the right hemisphere) found IED spikes in the right anterior temporal and anterior frontal regions. Based on the icEEG findings, the right anterior temporal region (length: $5 \mathrm{~cm}$ from the anterior temporal pole; size: $\sim 5 \times 3 \times 3 \mathrm{~cm}^{3}$ ) together with mesial temporal structures, such as the hippocampus and the amygdala (size: $\sim 1.5 \times 1.5 \times 1.5 \mathrm{~cm}^{3}$ ) were resected, and the right anterior frontal region (length: $6 \mathrm{~cm}$ from the anterior frontal pole; size: $\sim 6 \times 4 \times 2.5 \mathrm{~cm}^{3}$ ) was also resected. The findings of the eighth and ninth EEG-fMRI analyses were largely concordant with the combined EEG and icEEG findings.

The histopathological examination found dual pathology (coexistence of medial temporal sclerosis and extrahippocampal lesion) in the resected specimen: hippocampal sclerosis, and focal cortical dysplasia (type II). Postsurgical video EEG revealed that: (1) at 4 months postsurgery, IEDs in the right frontal and temporal regions were largely reduced, while prominent IEDs were in the left frontal region, and ictal spikes were more prominent in the left temporal region; and (2) at 16 months postsurgery, a large amount of IEDs in both hemispheres, especially in the left frontal region, and ictal spikes in both hemispheres, especially in the left frontal and temporal regions. The findings of postsurgical video EEGs reflected some basic facts: the patient was seizure-free for 3 months after surgery; seizure restarted after the AEDs were stopped (by the patient); and resuming AEDs did not stop seizure recurrence. The overall surgical outcome was between Engel class II and III. 
A

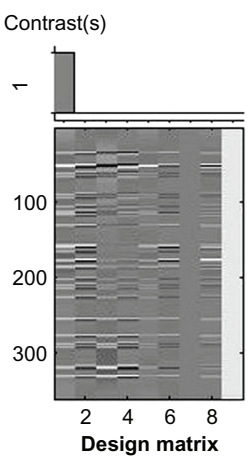

D

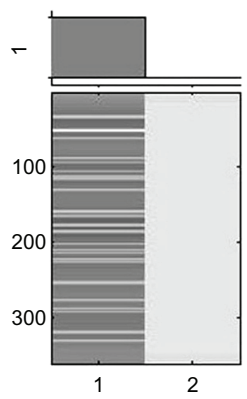

G

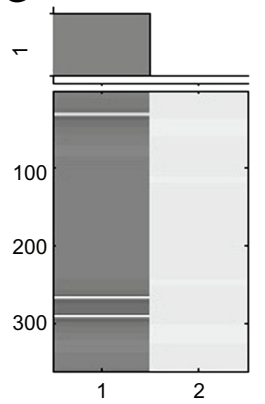

B

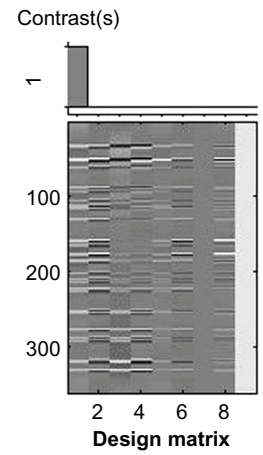

E

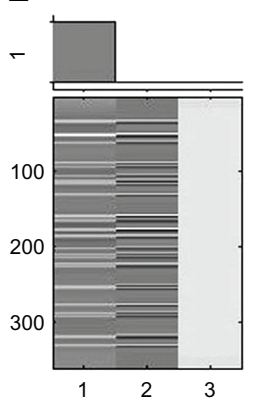

H

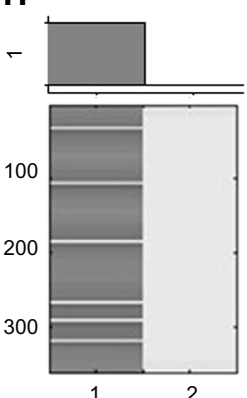

\section{C}

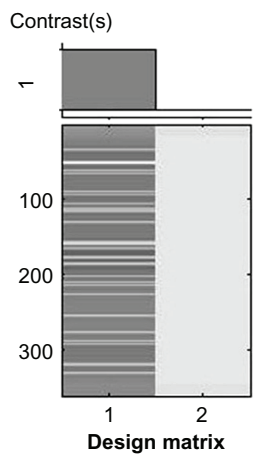

$\mathbf{F}$
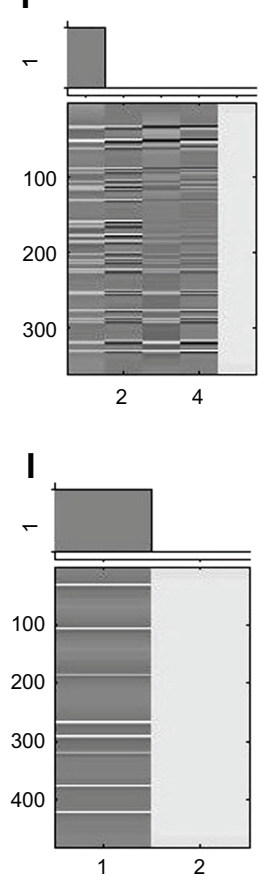

Figure 2 Design matrices for $\mathrm{fMRI}$ analyses with nine different sets of parameters. Notes: The design matrices (A-I) correspond to the analyses I-9, respectively, listed in Table I.

Abbreviation: $\mathrm{fMRI}$, functional magnetic resonance imaging.

\section{Discussion}

The EEG and recent EEG-fMRI findings indicated that this was a case of bi-TLE and ETLE with multiple seizure foci in the temporal and frontal regions of both hemispheres, and the seizure foci in the right temporal and frontal regions were confirmed by icEEG (which covered those regions).

\section{Lessons learned from EEG-fMRI analysis and the value of EEG-fMRI}

As a promising neuroimaging technique, the clinical value of EEG-fMRI in presurgical evaluation is of interest to clinicians. This study revealed that the failure of our previous

EEG-fMRI analysis may lie in two factors: inaccurate IED mark-up and inappropriate fMRI analysis.

First, inaccurate IED mark-up has a large impact on EEG-fMRI results. ${ }^{8}{ }^{817}$ In this study, IED onsets 1 and 2 included more nonepileptiform events than did onsets 3,4 , and 5 . When we compared the analyses that used the same model options and parameters but different IED onsets (the third and fourth analyses used onsets 1 and 2 while the seventh to ninth analyses used onsets 3-5), it seemed that including more nonepileptiform events in the IED onsets resulted in more voxels that survived a relatively low threshold $(\mathrm{T}=2.34)$ and a number of voxels that survived a higher threshold $(\mathrm{T}=4.73)$. This might be an exception to the results obtained by Flanagan et al. ${ }^{20}$ In their study $(\mathrm{n}=10)$, Flanagan et al found that one patient showed more voxels above threshold, while the other nine patients demonstrated fewer voxels above threshold, when epileptiform and nonepileptiform events were mixed in the SPM analysis, and they concluded that mixing epileptiform and nonepileptiform events generally (but not always) decreased the number of voxels that survived threshold. Thus, including nonepileptiform events in IED onsets may lead to misleading results (either more or fewer voxels that survived threshold). Similarly, omitting true epileptiform events or severely inconsistent event mark up may be misleading as well. ${ }^{20}$ Therefore, accurate IED mark up is critical for obtaining true IED-related activation in EEG-fMRI analysis. ${ }^{20,21}$ Further studies on accurate IED mark-up and more efficient IED identification, for example, semiautomatic and automatic IED identification, ${ }^{16,21-24}$ are needed in the future.

Second, this study demonstrated that the selection of SPM GLM model options in the fMRI analysis could have a large impact on EEG-fMRI results. Among the nine fMRI analyses (Table 1) that had different model options and parameters, the first analysis used the same set of model options and parameters and obtained the same results as those of our previous analysis. The key problem in this set of model options and parameters lies in the "model (Volterra) interactions"25 option. Volterra analysis is usually used in the event-related fMRI to model interactions between different stimulus (for example, visual and audio) events with certain conditions, especially when the stimulus events are very close to each other over time and interact with each other, especially when the interactions of the hemodynamic responses in the brain are nonlinear. ${ }^{25}$ However, the IED events in this case were very short (in the order of milliseconds) and widely separated in sequential scans $(\mathrm{TR}=2.56 \mathrm{sec})$. Thus, it is inappropriate 


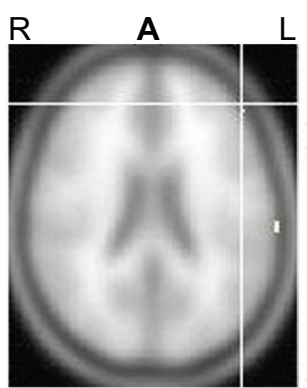

C
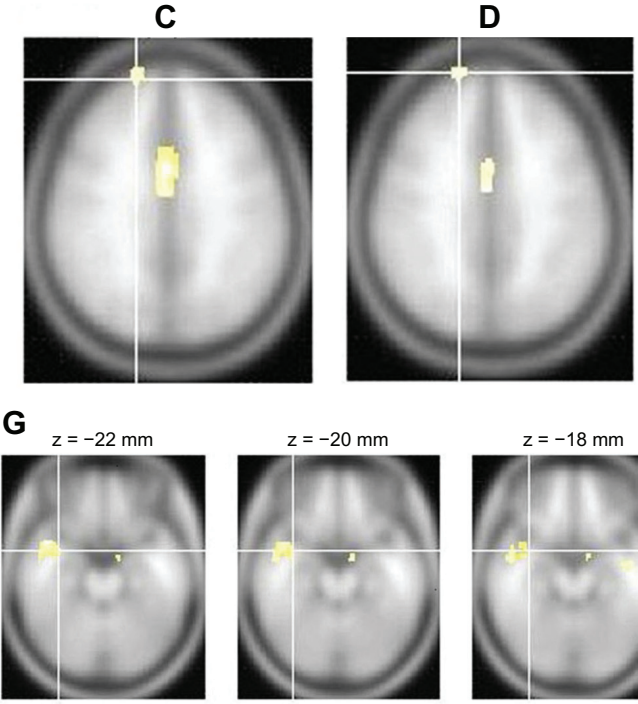

H $\quad z=-10 \mathrm{~mm}$

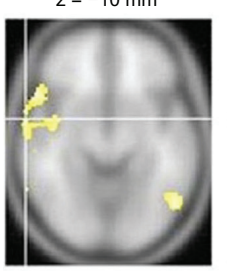

I $z=-8 \mathrm{~mm}$

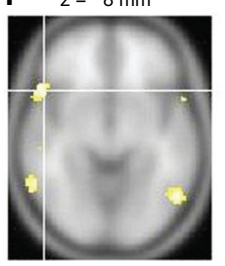

$z=-20 m m$

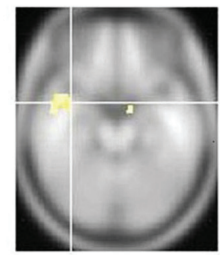

$z=-8 m m$

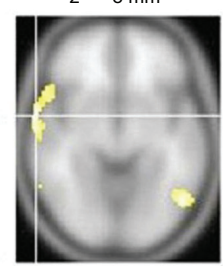

$z=-6 \mathrm{~mm}$

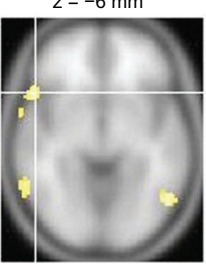

B

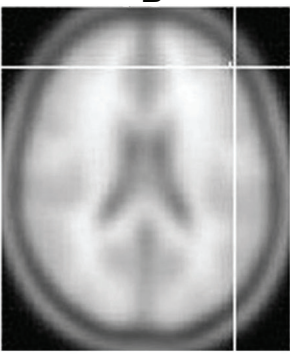

D

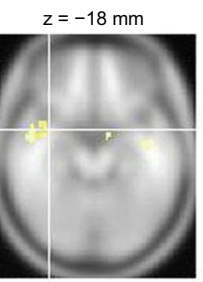

$z=-6 m m$

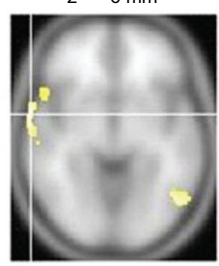

$z=-4 m m$

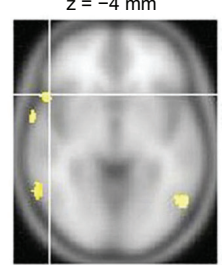

T value

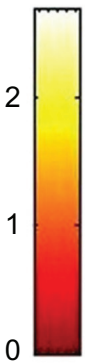

F
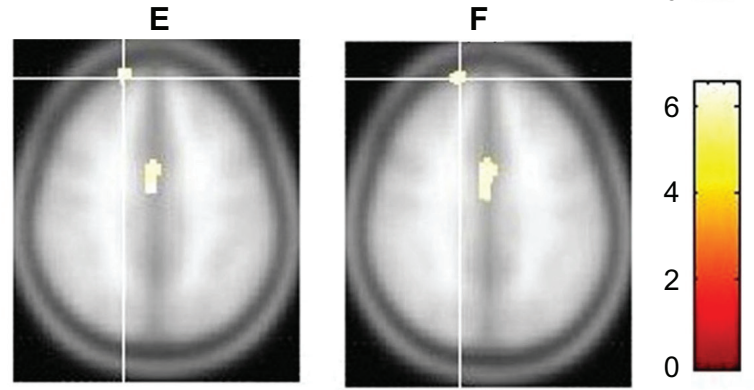

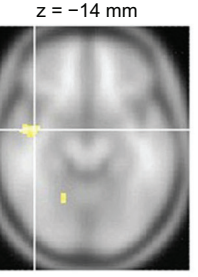

$\mathrm{z}=32 \mathrm{~mm}$

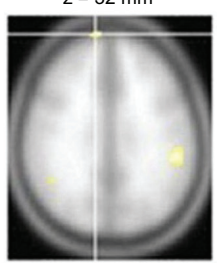

$z=30 \mathrm{~mm}$

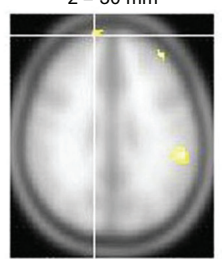

$z=-12 \mathrm{~mm}$

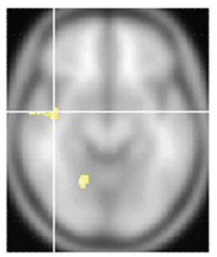

$z=34 \mathrm{~mm}$

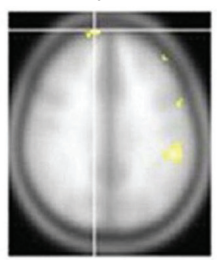

$z=32 \mathrm{~mm}$

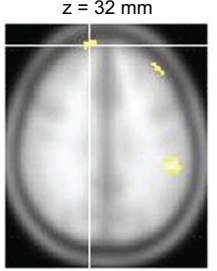

$z=-10 \mathrm{~mm}$

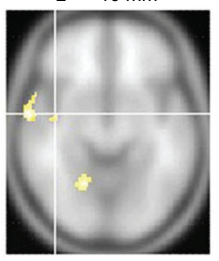

$z=36 \mathrm{~mm}$

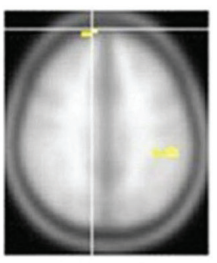

$\mathrm{z}=34 \mathrm{~mm}$

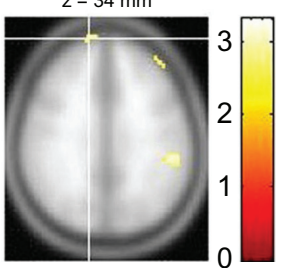

Figure 3 The results of the $\mathrm{PMRI}$ analyses.

Notes: The slice views (A-F) showing fMRI activation in the frontal regions correspond to analyses I-6, respectively, in Table I, while (G-I) correspond to the last analyses 7-9, respectively, in Table I and reveal activation in the temporal and/or frontal regions. The results of analyses $3-6$ are presented with a threshold at $T=4.73(P<0.05$ [FWE corrected]), while the results of analyses I,2 and 7-9 are presented with a threshold at $T=2.34(P<0.0$ I [unc]).

Abbreviations: FWE, family-wise multiple comparisons; L, left; R, right; unc, uncorrected for multiple comparisons; fMRI, functional magnetic resonance imaging.

to use the Volterra series to model interactions of the IED events in this case.

A number of studies have confirmed the colocalization of IEDs and fMRI activation. ${ }^{5,21}$ Although the generators of IEDs that EEG-fMRI detects are actually the irritative zone (IZ) (ie, the brain region that generates IED), rather than the epileptogenic zone (EZ) (ie, the seizure focus), ${ }^{26}$ the IZ is often located near the EZ or in the surrounding area of EZ. ${ }^{19}$ There is clinical value in detecting the IZ with EEG-fMRI in the presurgical evaluation of epilepsy, and EEG-fMRI often identifies distributed brain areas of interictal spiking in epileptic patients who have frequent IEDs. ${ }^{8,10,20}$ For example, Zijlmans et al have convincingly demonstrated that EEGfMRI could improve source localization and corroborate a 
negative decision regarding surgical candidacy in complex clinical cases (with either unclear focus or multifocality) and EEG-fMRI is a valuable tool in presurgical evaluation. ${ }^{11}$ Our recent EEG-fMRI findings were largely concordant with the combined findings of EEG and icEEG, which indicated that EEG-fMRI is valuable in presurgical evaluation, but it requires caution. Other neuroimaging tests, such as positron emission tomography (PET) and single photon emission tomography computerized (SPECT) may also be helpful in localizing the seizure foci, but their spatial resolution might not be as good as EEG-fMRI. A comparison of EEG-fMRI with PET or SPECT in focus localization could be explored in the future.

\section{Reasons for non-seizure-free outcome}

The non-seizure-free outcome of this case may be due to the complex bi-TLE and ETLE or multifocality in this patient. In the literature, five major causes of non-seizure-free outcome have been identified: (1) insufficient resection of mesial temporal structures; (2) insufficient or nonresection of the temporal neocortex; (3) dual pathology (coexistence of mesial temporal sclerosis and a neocortical lesion); (4) relapse on the contralateral temporal lobe; and (5) extratemporal and temporal plus epilepsy. ${ }^{2}$ In this study, the findings of EEG and EEG-fMRI, together with the non-seizure-free outcome, revealed the multifocal nature of this case: Removal of the seizure foci in the right hemisphere did improve the patient's condition (the patient was seizure free for 3 months); while the seizure foci in the left hemisphere were left intact and still generated IEDs and ictal spikes over time (as indicated by postsurgical EEG), and caused seizure recurrence (contralateral relapse).

In addition, the dual pathology in the right temporal and frontal regions, which was likely the coexistence of hippocampal sclerosis and cortical dysplasia, may make this case more complicated. Identifying lesions with dual pathology is challenging because the subtle signs of medial temporal sclerosis (or hippocampal sclerosis) and mild changes of dysplasia can be missed on presurgical MRI or by other neuroimaging techniques. ${ }^{2}$ In this case, the seizure foci in the right hemisphere were detected and confirmed by intraoperative icEEG, and their removal in the right anterior temporal and frontal regions seemed to be effective in achieving the transient seizure-free outcome in this patient. However, the seizure foci in the left hemisphere not only existed, but also may have had dual pathology. Currently, this patient must take several AEDs for seizure control. It is possible that the seizures will become drug resistant again and that the medications will become less effective, but the patient will still have to take them for the rest of his life. Alternatively, reoperation may be considered. Reoperation has been applied to a number of patients with drug-resistant focal epilepsy who underwent ineffective epilepsy surgery. ${ }^{1,2}$ However, reoperation may not be a good option for this case because important eloquent cortices such as the language cortex are closely mingled with the seizure foci, in the left frontal and temporal regions. Surgery in such regions could lead to incomplete/insufficient resection and thus reoperation failure. Vagus nerve stimulation (VNS) might be considered instead in this case. Further study with better diagnostic and treatment techniques in bi-TLE and ETLE (with dual pathology) is needed to improve the treatment of patients with similar conditions.

\section{Acknowledgments}

We are grateful to He Shaodong and Zhang Xingang at Shenzhen Hanxiang Biomedical Ltd., for the EEG system; to Zhuo Yan, Wang Bo, and Liu Zhendong at Beijing MRI Brain Imaging Center for data acquisition; and to Wang Zhengge at Nangjing Hospital and Zhan Zhichao at Beijing Normal University, for the IED mark-up. This study was partially supported by the Natural Science Foundation of the People's Republic of China (grant number 81071211).

\section{Disclosure}

The authors report no conflicts of interest in this work.

\section{References}

1. Téllez-Zenteno JF, Dhar R, Wiebe S. Long-term seizure outcomes following epilepsy surgery: a systematic review and meta-analysis. Brain. 2005;128(Pt 5):1188-1198.

2. Harroud A, Bouthillier A, Weil AG, Nguyen DK. Temporal lobe epilepsy surgery failures: a review. Epilepsy Res Treat. 2012;2012:201651.

3. Rosenkranz K, Lemieux L. Present and future of simultaneous EEG-fMRI. MAGMA. 2010;23(5-6):309-316.

4. Al Asmi A, Bénar CG, Gross DW, et al. fMRI activation in continuous and spike-triggered EEG-fMRI studies of epileptic spikes. Epilepsia. 2003;44(10):1328-1339.

5. Bagshaw AP, Aghakhani Y, Bénar CG, et al. EEG-fMRI of focal epileptic spikes: analysis with multiple haemodynamic functions and comparison with gadolimium-enhanced MR angiograms. Hum Brain Mapp. 2004;22(3):179-192.

6. Bénar CG, Grova C, Kobayashi E, et al. EEG-fMRI of epileptic spikes: concordance with EEG source localization and intracranial EEG. Neuroimage. 2006;30(4):1161-1170.

7. Gotman J, Kobayashi E, Bagshaw AP, Bénar CG, Dubeau F. Combining EEG and fMRI: a multimodal tool for epilepsy research. J Magn Reson Imaging. 2006;23(6):906-920.

8. Gotman J. Epileptic networks studied with EEG-fMRI. Epilepsia 2008;49 Supp1 3:S42-S51.

9. Salek-Haddadi A, Diehl B, Hamandi K, et al. Hemodynamic correlates of epileptiform discharges: and EEG-fMRI study of 63 patients with focal epilepsy. Brain Res. 2006;1088(1):148-166.

10. Krakow K. Imaging epileptic activity using functional MRI Neurodegener Dis. 2008;5(5):286-295. 
11. Zijlmans M, Huiskamp G, Hersevoort M, Seppenwoolde JH, van Huffelen AC, Leijten FS. EEG-fMRI in the preoperative work-up for epilepsy surgery. Brain. 2007;130(Pt 9):2343-2353.

12. De Tiège $X$, Laufs $H$, Boyd $S G$, et al. EEG-fMRI in children with pharmacoresistant focal epilepsy. Epilepsia. 2007;48(2):385-389.

13. Grova C, Daunizeau J, Kobayashi E, et al. Concordance between distributed EEG source localization and simultaneous EEG-fMRI studies of epileptic spikes. Neuroimage. 2008;39(2):755-774.

14. Tyvaert L, Hawco C, Kobayashi E, LeVan P, Dubeau F, Gotman J. Different structures involved during ictal and interictal epileptic activity in malformations of cortical development: an EEG-fMRI study. Brain. 2008;131(Pt 8):2042-2060.

15. Moeller F, Tyvaert L, Nguyen DK, et al. EEG-fMRI: adding to standard evaluations of patients with nonlesional frontal lobe epilepsy. Neurology. 2009;73(23):2023-2030.

16. Thornton RC, Rodionov R, Laufs $\mathrm{H}$, et al. Imaging haemodynamic changes related to seizures: comparison of EEG-based general linear model, independent component analysis of $\mathrm{fMRI}$ and intracranial EEG. Neuroimage. 2010;53(1):196-205.

17. Grouiller F, Thornton RC, Groening K, et al. With or without spikes: localization of focal epileptic activity by simultaneous electroencephalography and functional magnetic resonance imaging. Brain. 2011; 134(Pt 10):2867-2886.
18. Zhang J, Liu W, Chen H, et al. EEG-fMRI validation studies in comparison with icEEG: a review. Int J Psychophysiol. 2012;84(3):233-239.

19. Rosenow F, Lüders H. Presurgical evaluation of epilepsy. Brain. 2001;124(Pt 9):1683-1700.

20. Flanagan D, Abbott DF, Jackson GD. How wrong can we be? The effect of inaccurate mark-up of EEG/fMRI studies in epilepsy. Clin Neurophysiol. 2009;120(9):1637-1647.

21. Kobayashi E, Bagshaw AP, Bénar CG, et al. Temporal and extratemporal BOLD responses to temporal lobe interictal spikes. Epilepsia. 2006;47(2):343-354.

22. Liston AD, De Munck JC, Hamandi K, et al. Analysis of EEG-fMRI data in focal epilepsy based on automated spike classification and Signal Space Projection. Neuroimage. 2006;31(3):1015-1024.

23. Formaggio E, Storti SF, Bertoldo A, Manganotti P, Fiaschi A, Toffolo GM. Integrating EEG and fMRI in epilepsy. Neuroimage. 2011;54(4):2719-2731.

24. Jann K, Wiest R, Hauf M, et al. BOLD correlates of continuously fluctuating epileptic activity isolated by independent component analysis. Neuroimage. 2008;42(2):635-648.

25. Friston KJ, Josephs O, Rees G, Turner R. Nonlinear event-related responses in fMRI. Magn Reson Med. 1998;39(1):41-52.

26. Bernasconi A, Bernasconi N, Bernhardt BC, Schrader D. Advances in MRI for 'cryptogenic' epilepsies. Nat Rev Neurol. 2011;7(2):99-108.
Neuropsychiatric Disease and Treatment

\section{Publish your work in this journal}

Neuropsychiatric Disease and Treatment is an international, peerreviewed journal of clinical therapeutics and pharmacology focusing on concise rapid reporting of clinical or pre-clinical studies on a range of neuropsychiatric and neurological disorders. This journal is indexed on PubMed Central, the 'PsycINFO' database and CAS.

\section{Dovepress}

The manuscript management system is completely online and includes a very quick and fair peer-review system, which is all easy to use. Visit http://www.dovepress.com/testimonials.php to read real quotes from published authors. 